\title{
The Generation Problem of Assertion and Query Algorithm Based on Context Logic Reasoning to Implement
}

\author{
shexuebing \\ Jiangxi Institute of Technology \\ Department of digital technology Nanchang, China \\ shexuebing1982@sohu.com
}

\begin{abstract}
.
the study of natural language from birth has become an important field of artificial intelligence. Natural language will become an important way of human-computer interaction. In some specific occasions, often use language refers to various objects, to make assertions about their problems, etc.. Refer to the problem because of the certainty and uncertainty, so that the complexity of the problem. This paper will establish a database management system, the system uses natural language interface technology to realize the anaphora assertions and queries.
\end{abstract}

Keywords: Llogic reasoning;refer to problem;assertion;query algorithm

\section{Introduction}

To recognize speech acts, we must also consider the sentence structure and language environment, through the context to eventually come to a right understanding of language. In order to have a general method to explain the language behavior, often need to express different multi-agent belief system, and the system can be based on the knowledge of the various explanations for screening. For example: the intelligent body if the "Do you know the time? " as a condition of intention, it must know the time; on the other hand, if this sentence as a problem, then it must not know time. Through the application of conditional check words, can remove many inappropriate interpretation. Intelligent systems usually explain the problem of speech acts as a kind of plan recognition problem. The system tries to identify the speaker's intentions, and will meet the prophet to interpret the behavior of selected for the intended interpretation. 
Composition of predicate formulas knowledge base is mainly composed of constant and the expression domain of facts. Application of anaphora refers to the knowledge base to identify noun phrase refers to the generation of the constant. There are four types of noun phrases to note: description of proper nouns, pronouns, definite and indefinite NOUN phrases[1].

Treatment of proper nouns usually simple, generally assumes that the system has the object with the name in the domain of the corresponding knowledge. Therefore, to explain is the proper name, system as long as the lookup table can be.

The pronoun usually refers to the object I just mentioned. The simplest method is to establish pronoun processing history table records, all the objects mentioned above. These objects are sorted, object recently mentioned will be preferred. A simple method for treatment of pronoun is the first object to find the characteristics and the pronoun in the history table to match, the general is the right answer[2]. For example, consider the following three sentences in dialogue:

1a. Tom went to the store yesterday

$1 \mathrm{~b}$. The manager was very rude to the customers

1c. He insulted them

In the sentence $1 \mathrm{C}$ he pronoun should refer to the history table singular masculine ( instead of customers ), he and manager may be referred to the sentences in 1b. Although Tom is also possible, but manager was recently mentioned. The pronoun "them" refers to the customers. The deterministic description usually refers to the object history table, even if it can not only describe the objects in the world, also can successfully complete a generation. Consider the following dialogue:

2a. I bought a pencil and a pen at the store

2b. The pen did not work

In the sentence $2 \mathrm{~B}$, deterministic description the pen refers to the sentence in the $2 \mathrm{~A}$ pen, although there are a lot of pen in the knowledge base. In addition, the deterministic description page can refer to not mentioned earlier in the knowledge base only object.

Uncertainty description ( such as a pen ) is usually used to the new object extraction in conversation, this can be through the knowledge base to build a new constant and known in the assertion information processing[3]. So, for uncertain a pen, system and build a new constant X33, and the ( PEN X33 ) is added to the database and the history tables, the object in the future by deterministic description refers to the generation of sentences, such as $2 \mathrm{~b}$. However, method for processing non deterministic description method and processing in the above questions are different. Assume that the knowledge base contains assertions ( OWNS JACK1 PENCIL1 ) and ( PENCIL PENCIL1), consider the question:

3. Does Jack own a pencil?

The assumption that the uncertainty in front of the description methods will produce new constant X34 and ( PEN X34 ) is added to the database, then the query OWNS JACK1 X34 will fail, because the database does not exist in the knowledge of Jack owns X34. This and in logic of existential quantifiers are very 
similar to Skolem standardization. In the processing of an assertion, the use of Skolem standardization is a correct way, but when processing a query, the existential quantifier must be mapped to a variable. The 3 sentences should be

(OWNS JACK1(? x) (PENCIL (? x)))

\section{Process database assertions and queries}

The establishment of a database management system, the system uses natural language interface technology to realize the assertions and queries. The database system provides two functions: add Assert notification table to a database, Query database to search and return all possible bindings associated with the current query set. Here are some typical database interoperability.

Assert (P A B) adds( $\mathrm{P} \mathrm{A} \mathrm{B}$ ) to the database

Assert (P A C) adds( $\mathrm{P}$ A C) to the database

Assert (Q C) adds( $Q$ C) to the database

Query (P A (? x)) returns two answers, one with(? x)bound to A, and the other with (? $\mathrm{x})$ bound to $\mathrm{C}$

Query (AND(P A ? x)(Q (? x))) returns one answers, with(? $\mathrm{x})$ bound to $\mathrm{C}$

Write processing refer to and interaction of the new code and the parser, you can create a simple natural language interface for database. A given grammar and vocabulary, the parser can handle various semantic form includes the speech act operator. The following assertions and query processing given extended grammar and vocabulary[4].

1) (S (SEM (assert-prop (? sem-vp)) ) (AGR (? a))

(NP (SEM (? sem-np)) (AGR (? a)))

(VP (SEM (? sem-np) (SUBJ (? sem-np)) (AGR (? a)))

2) (S (SEM (y-n-query (? sem-vp)) ) (AGR (?a) )

(AUX (SEM (? sem-aux )) )

(NP (SEM (? sem-np ) ) (AGR (? a)) )

(VP (SEM (? sem-np) (SUBJ (? sem-np)) (AGR (? a) ) (VFORM base)

3) (NP ( SEM ( (? sem-art) *( (? sem-n $) *))$ (AGR (? a ) )

(ART (SEM (? sem-art ) ) (AGR (? a)) )

( N (SEM (? sem-n)) (AGR (? a)) )

4) (NP ( SEM ( (? sem-art)* ( (? sem-n $) *)((?$ sem-adj $) *)$

(AGR (?a) ) )

(ART (SEM (? sem-art ) ) (AGR (?a)))

(ADJ (SEM (? sem-adj) ) )

( N (SEM (? sem-n)) (AGR (? a) ) ) 


\section{5) (NP (SEM (PRO* ( (? sem-pro)*) ) (AGR (? a ) ) ) (PRO (SEM (? sem-pro)) (AGR (? a)))}

A description will be converted into usable form. Processing method for deterministic description is first check whether the description of object in history, and if by chance there is only one answer, to determine the logical form of replacement in description on the use of the object; if there is no such object; if there is no such object, check all the object description satisfies the query database, if this is only one answer, also use it to replace; if there is still no or more than one answer, it can be considered that the sentence is not complete[5]. Processing of pronouns is similar, only the search history table, in a history table in check, find the nearest object pronouns can meet the constraint condition. Uncertainty description can only appear in assertions. Assertion is with declarative properties of the new constant is added to the database.

\section{Arithmetic statement}

The following algorithm is used to process the certainty, uncertainty description and pronoun. The deterministic description ( The $\mathrm{x}(\mathrm{P} \mathrm{x})$ ) of the logical form:

1) to meet the search in the history table ( P (? X ) ) the nearest object conditions, if there is only one solution, then it is returned as the value;

2) the search in the database satisfy ( $P($ ? The variables ( $\mathrm{x})$ )? X ), object ANS is returned by the query;

3) if a value only containing ANS, then the update history table, and take it as the return value;

4) otherwise, failure

Given the uncertain description ( $\mathrm{A} \times(\mathrm{P} \mathrm{x})$ ) of the logical form:

1) if the expression in the assertion of, respectively, to create a new $X$ object and assertions in the database ( $\mathrm{P} \mathrm{x}$ ), modify the history table, take $\mathrm{X}$ as the return value.

Given the pronouns ( $\mathrm{PRO} x(\mathrm{P} \mathrm{x})$ ) of the logical form:

2) to meet the search in the history table ( $\mathrm{P} \mathrm{O}$ ) is really the nearest object O.

The function defined above, then define a truncated form of logic function used to process each noun phrase is simple.

\section{Summary}

Many natural language systems are the logical form for solving the characteristics of sentence making in syntactic analysis and application of the completion of the initial explanation. Processing context requires commonsense knowledge. This paper solves the problem of deterministic description refers to the technology, explains how to realize a simple interface and database management system of natural language system. 


\section{References}

[1] Cai Zixing Xu Guangyou " artificial intelligence and Applications " Tsinghua University press, 2011 April Edition

[2] Thomas Dean, James Allen " artificial intelligence and practice of " Electronic Industry Press in 2009 April Edition

[3]Aarup,M; Arentoft,M.M.:Parrod,Y; OPTIMUM-AIV:A knowledge based planning and scheduling system for spacecraft AIV 2008

[4] Abelson,Haold and Sussman,Gerald Jay 2007.Structure and Interpretation of Computer Programs. MIT Press,Cambridge,Massachusetts.

[5] Allen,James F.;Hendler ,James;and Tade,Austin,editors 2007.Redings in planning.Morgan Kaufmann,san mateo,California. 